\title{
Exponential Filtering for Uncertain Markovian Jump Time-Delay Systems With Nonlinear Disturbances
}

\author{
Zidong Wang, Senior Member, IEEE, James Lam, Senior Member, IEEE, and Xiaohui Liu
}

\begin{abstract}
In this paper, we study the robust exponential filter design problem for a class of uncertain time-delay systems with both Markovian jumping parameters and nonlinear disturbances. The jumping parameters considered here are generated from a continuous-time discrete-state homogeneous Markov process, and the parameter uncertainties appearing in the state and output equations are real, time dependent, and norm bounded. The time-delay and the nonlinear disturbances are assumed to be unknown. The purpose of the problem under investigation is to design a linear, delay-free, uncertainty-independent state estimator such that, for all admissible uncertainties as well as nonlinear disturbances, the dynamics of the estimation error is stochastically exponentially stable in the mean square, independent of the time delay. We address both the filtering analysis and synthesis issues, and show that the problem of exponential filtering for the class of uncertain time-delay jump systems with nonlinear disturbances can be solved in terms of the solutions to a set of linear (quadratic) matrix inequalities. A numerical example is exploited to demonstrate the usefulness of the developed theory.
\end{abstract}

Index Terms-Linear matrix inequalities (LMIs), Markovian jump systems, nonlinear disturbances, robust filtering, time delay.

\section{INTRODUCTION}

$\mathbf{T}$ HE well-known Kalman filtering approach assumes that the system model is well-posed and its disturbances are Gaussian noises with known statistics. Therefore, Kalman filters can be sensitive to system data and have poor performance robustness when a good system model is hard to obtain or the system drifts. Motivated by this problem, the research on robust filtering has been very attractive in the past decade and many results have been obtained with respect to various filtering performance criteria, see [2], [5], and references therein.

On the other hand, many physical systems have variable structures subject to random abrupt changes, which may result from abrupt phenomena such as random failures and repairs of the components, changes in the interconnections of subsystems, sudden environmental changes, modification of the operating point of a linearized model of a nonlinear systems, etc. A system with this character may be modeled as a hybrid one;

Manuscript received March 25, 2002; revised August 10,2002. This work was supported in part by the Engineering and Physical Science Research Councitl (EPSRC) under Grant GR/S27658/01 and Grant GR/R35018/01, in part by the University of Hong Kong under HKU CRCG Grant 10203795, in part by the Nuffield Foundation under Grant NAL/00630/G, and in part by the Alexander von Humboldt Foundation of Germany. This paper was recommended by Associate Editor X. Yu.

Z. Wang and X. Liu are with the Department of Information Systems and Computing, Brunel University, Uxbridge, Middlesex UB8 3PH, U.K. (e-mail: Zidong.Wang@brunel.ac.uk).

J. Lam is with the Department of Mechanical Engineering, The University of

Hong Kong, Hong Kong.

Digital Object Identifier 10.1109/TCSII.2004.825596 that is, the state space of the system contains both discrete and continuous states. A special class of hybrid systems are the so-called jump linear systems (JLSs) which have attracted a lot of research interest since the mid 1960s. In the past decade, the optimal regulator, controllability, observability, stability and stabilization problems have been extensively studied for JLSs, see [2], [11] and references therein. Also, the filtering problem of systems with jumping parameters has recently begun to gain initial attention. Shi et al. [11] have studied the robust Kalman filtering problem for linear jump systems and have shown that the problem is solvable if two sets of coupled algebraic Riccati equations have positive definite solutions.

It is now well recognized that the dynamic behavior of many industrial processes contains inherent time delays and time delays are very often the cause for instability and poor performance of systems. In the past few years, a great many of papers have appeared on this general topic of robust and/or $H_{\infty}$ controller design problems for linear uncertain time-delay systems, see, e.g., [8] for a survey. In the filtering case, the robust Kalman filter design problem has been investigated in [2] and [5] for linear continuous and discrete time-delay systems. Unfortunately, in [2] and [5], only the asymptotical stability has been considered on the filtering process and, therefore, a possibly long convergence time may lead to poor performance. The exponential filtering problem has been recently studied in [12] for a class of nonlinear time-delay systems. It should be pointed out that, to date, there have been very few papers tackling the $e x$ ponential filter design problem for Markovian jump time-delay systems, not to mention the case where parameter uncertainty and nonlinear disturbance also exist in the system model. This situation motivates our present investigation.

This paper is concerned with the exponential filtering problem for a class of continuous time-delay uncertain systems with Markovian jumping parameters and nonlinear disturbances. We aim at designing a robust filter such that, for all admissible uncertainties as well as nonlinear disturbances, the dynamics of the estimation error of each system mode is stochastically exponentially stable in the mean square, independent of the time delay. We show that both the filter analysis and the filter synthesis problems can be tackled in terms of the solutions to a set of linear matrix inequalities (LMIs) (see [3]) or quadratic matrix inequalities (QMIs) (see [9]). We demonstrate the usefulness and applicability of the developed theory by means of a numerical example.

\section{A. Notation}

The notations in this paper are quite standard. $\mathbb{R}^{n}$ and $\mathbb{R}^{n \times m}$ denote, respectively, the $n$-dimensional Euclidean space 
and the set of all $n \times m$ real matrices. The superscript " $T$ " denotes the transpose and the notation $X \geq Y$ (respectively, $X>Y$ ) where $X$ and $Y$ are symmetric matrices, means that $X-Y$ is positive semi-definite (respectively, positive definite). $I$ is the identity matrix with compatible dimension. We let $h>0$ and $C\left([-h, 0] ; \mathbb{R}^{n}\right)$ denote the family of continuous functions $\varphi$ from $[-h, 0]$ to $\mathbb{R}^{n}$ with the norm $\|\varphi\|=\sup _{-h \leq \theta \leq 0}|\varphi(\theta)|$, where $|\cdot|$ is the Euclidean norm in $\mathbb{R}^{n}$. If $A$ is a real matrix, we denote by $\|A\|$ its operator norm, i.e., $\|A\|=\sup \{|A x|:|x|=1\}=\sqrt{\lambda_{\max }\left(A^{T} A\right)}$, where $\lambda_{\max }(\cdot)$ [respectively, $\left.\lambda_{\min }(\cdot)\right]$ means the largest (respectively, smallest) eigenvalue of $A . l_{2}[0, \infty]$ is the space of square integrable vector. Moreover, let $\left(\Omega, \mathcal{F},\left\{\mathcal{F}_{t}\right\}_{t>0}, P\right)$ be a complete probability space with a filtration $\left\{\mathcal{F}_{t}\right\}_{t>0}$ satisfying the usual conditions (i.e., the filtration contains all $P$-null sets and is right continuous). Denote by $L_{\mathcal{F}_{0}}^{p}\left([-h, 0] ; \mathbb{R}^{n}\right)$ the family of all $\mathcal{F}_{0}$-measurable $C\left([-h, 0] ; \mathbb{R}^{n}\right)$-valued random variables $\xi=\{\xi(\theta):-h \leq \theta \leq 0\}$ such that $\sup _{-h \leq \theta \leq 0} \mathbb{E}|\xi(\theta)|^{p}<\infty$, where $\mathbb{E}\{\cdot\}$ stands for the mathematical expectation operator with respect to the given probability measure $P$.

\section{PROBLEM FORMULATION AND ASSUMPTIONS}

Let $\{r(t), t \geq 0\}$ be a right-continuous Markov process on the probability space which takes values in the finite space $\mathcal{S}=\{1,2, \ldots, N\}$ with generator $\Pi=\left(\gamma_{i j}\right)(i, j \in \mathcal{S})$ given by

$$
P\{r(t+\Delta)=j \mid r(t)=i\}= \begin{cases}\gamma_{i j} \Delta+o(\Delta), & \text { if } i \neq j \\ 1+\gamma_{i i} \Delta+o(\Delta), & \text { if } i=j\end{cases}
$$

where $\Delta>0$ and $\lim _{\Delta \rightarrow 0} O(\Delta) / \Delta=0, \gamma_{i j} \geq 0$ is the transition rate from $i$ to $j$ if $i \neq j$ and $\gamma_{i i}=-\sum_{j \neq i} \gamma_{i j}$.

In this paper, we consider a class of uncertain continuous-time state delayed jump systems with nonlinear disturbances described by

$$
\begin{aligned}
\dot{x}(t)= & {[A(r(t))+\Delta A(t, r(t))] x(t)+A_{d}(r(t)) x(t-h) } \\
& +D(r(t)) f(x(t, r(t))) \\
x(t)= & \varphi(t) \\
r(t)= & r(0), \quad t \in[-h, 0] \\
y(t)= & {[C(r(t))+\Delta C(t, r(t))] x(t) }
\end{aligned}
$$

where $x(t) \in \mathbb{R}^{n}$ is the state, $y(t) \in \mathbb{R}^{p}$ is the measurement output, $f(\cdot): \mathbb{R}^{n} \rightarrow \mathbb{R}^{n_{f}}$ is an unknown nonlinear exogenous disturbance input, $h$ denotes the constant, bounded, but unknown state delay, $\varphi(t)$ is a continuous vector valued initial function. For a fixed system mode, $A(r(t)), A_{d}(r(t)), D(r(t))$, and $C(r(t))$ are known constant matrices with appropriate dimensions. $\Delta A(t, r(t))$ and $\Delta C(t, r(t))$ are real-valued matrix functions representing norm-bounded parameter uncertainties and satisfy

$$
\left[\begin{array}{c}
\Delta A(t, r(t)) \\
\Delta C(t, r(t))
\end{array}\right]=\left[\begin{array}{l}
M_{1}(r(t)) \\
M_{2}(r(t))
\end{array}\right] F(t, r(t)) N(r(t))
$$

where for a fixed system mode, $M_{1}(r(t)), M_{2}(r(t))$, and $N(r(t))$ are known real constant matrices of appropriate dimensions which characterize how the deterministic uncertain parameter in $F(t, r(t))$ enters the nominal matrix $A(r(t))$ and $C(r(t))$, and $F(t, r(t))$ is an unknown time-varying matrix function meeting

$$
F^{T}(t, r(t)) F(t, r(t)) \leq I, \forall t \geq 0 ; r(t)=i \in \mathcal{S}
$$

The uncertainties $\Delta A(r(t)), \Delta C(r(t))$ are said to be admissible if both (4) and (5) are satisfied.

Remark 1: We point out that the exogenous nonlinear time-varying disturbance term $f(x(t, r(t)))$ in the system model (1)-(3) has not been taken into account in the research literature concerning jump systems. Such a kind of disturbances may result from the linearization process of an originally highly nonlinear plant or may be actual external nonlinear inputs.

Remark 2: A more general case is that the external disturbances appear in both the system equation and the output measurement equation and one of the design objectives is to guarantee a given disturbance rejection attenuation level for the filtering process, in terms of a $H_{\infty}$-norm for the nonlinear timedelay systems. This gives a significant topic for future research.

Assumption 1: The system matrix $A(r(t))(\forall r(t)=i \in \mathcal{S})$ is Hurwitz stable. That is, for each $r(t)=i \in \mathcal{S}$, all eigenvalues of $A(i)$ are located in the left-half complex plane.

Assumption 2: The matrix $M_{2}(r(t))(\forall r(t)=i \in \mathcal{S})$ is of full row rank.

Assumption 3: For a fixed system mode, there exists a known real constant matrix $H(r(t)) \in \mathbb{R}^{n \times n}$ such that the unknown nonlinear vector function $f(\cdot)$ satisfies the following boundedness condition:

$$
|f(x(t, r(t)))| \leq|H(r(t)) x(t)| \quad \forall x(t, r(t)) \in \mathbb{R}^{n} .
$$

In this paper, the linear filter under consideration is of the following structure:

$$
\dot{\hat{x}}(t)=G(r(t)) \hat{x}(t)+K(r(t)) y(t)
$$

where $\hat{x}(t)$ is the state estimate, and for a fixed system mode, the constant matrices $G(r(t))$ and $K(r(t))$ are filter parameters to be designed.

Recall that the Markov process $\{r(t), t \geq 0\}$ takes values in the finite space $\mathcal{S}=\{1,2, \ldots, N\}$. For the sake of simplicity, we write

$$
\begin{aligned}
A(i):= & A_{i}, \quad A_{d}(i):=A_{d i}, \quad D(i):=D_{i} \\
C(i):= & C_{i}, \quad M_{1}(i):=M_{1 i}, \quad M_{2}(i):=M_{2 i} \\
N(i):= & N_{i}, \Delta A(t, i)=\Delta A_{i}(t), \quad \Delta C(t, i)=\Delta C_{i}(t) \\
F(t, i)= & F_{i}(t), H(i):=H_{i}, G(i):=G_{i}, K(i):=K_{i}, \\
& i \in \mathcal{S} .
\end{aligned}
$$

Now, we shall work on the system mode $r(t)=i, \quad \forall i \in \mathcal{S}$. Let the error state be

$$
e(t)=x(t)-\hat{x}(t)
$$

Then, it follows from (1) to (3) and (7) that

$$
\begin{aligned}
\dot{e}(t)= & G_{i} e(t)+\left[\left(A_{i}+\Delta A_{i}(t)\right)-K_{i}\left(C_{i}+\Delta C_{i}(t)\right)\right. \\
& \left.-G_{i}\right] x(t)+A_{d i} x(t-h)+D_{i} f(x(t, i)) .
\end{aligned}
$$


Moreover, we define

$$
\begin{aligned}
x_{f}(t) & :=\left[\begin{array}{l}
x(t) \\
e(t)
\end{array}\right] \\
A_{f i} & :=\left[\begin{array}{cc}
A_{i} & 0 \\
A_{i}-G_{i}-K_{i} C_{i} & G_{i}
\end{array}\right] \\
A_{f d i} & :=\left[\begin{array}{ll}
A_{d i} & 0 \\
A_{d i} & 0
\end{array}\right] \\
D_{f i} & :=\left[\begin{array}{l}
D_{i} \\
D_{i}
\end{array}\right], M_{f i}:=\left[\begin{array}{c}
M_{1 i} \\
M_{1 i}-K_{i} M_{2 i}
\end{array}\right] \\
N_{f i} & :=\left[\begin{array}{ll}
N_{i} & 0
\end{array}\right] \\
\Delta A_{f i}(t) & :=M_{f i} F_{i}(t) N_{f i} \\
F_{f} & :=\left[\begin{array}{ll}
I & 0
\end{array}\right] .
\end{aligned}
$$

Then, combining (1)-(3), (4), and (12) gives the following augmented system:

$$
\begin{aligned}
\dot{x}_{f}(t)=\left[A_{f i}+\Delta A_{f i}(t)\right] x_{f}(t)+ & A_{f d i} x_{f}(t-h) \\
& +D_{f i} f\left(F_{f} x_{f}(t, i)\right) .
\end{aligned}
$$

Observe the system (16) and let $x_{f}(t ; \xi)$ denote the state trajectory from the initial data $x_{f}(\theta)=\xi(\theta)$ on $-h \leq \theta \leq$ 0 in $L_{\mathcal{F}_{0}}^{2}\left([-h, 0] ; \mathbb{R}^{2 n}\right)$. Clearly, (16) admits a trivial solution $x_{f}(t ; 0) \equiv 0$ corresponding to the initial data $\xi=0$.

Definition 1: For the uncertain time-delay jump system (16) and every $\xi \in L_{\mathcal{F}_{0}}^{2}\left([-h, 0] ; \mathbb{R}^{2 n}\right)$, the trivial solution is exponentially stable in the mean square if, for every system mode, there exist scalars $\alpha>0$ and $\beta>0$ such that

$$
\mathbb{E}\left|x_{f}(t ; \xi)\right|^{2} \leq \alpha e^{-\beta t} \sup _{-h \leq \theta \leq 0} \mathbb{E}|\xi(\theta)|^{2} .
$$

The aim of this paper is to design a filter for the uncertain time-delay system with nonlinear disturbances in (1)-(3). We intend to design the filter parameters, $G_{i}$ and $K_{i}$, such that for all admissible time-varying parameter uncertainties $\Delta A_{f i}(t)$ and the nonlinear disturbance input $f(x(t, i))$, the augmented system (16) (and, therefore, the error dynamics) is exponentially stable in the mean square, independent of the unknown time delay $h$.

\section{MAIN RESUlTS AND PROOFS}

Lemma 1: (See, e.g., [11]) Let $M, N$, and $F$ be real matrices of appropriate dimensions with $F^{T} F \leq I$. Then, for any scalar $\mu \neq 0$, we have $M F N+N^{T} F^{T} M^{T} \leq \mu^{2} M M^{T}+\mu^{-2} N^{T} N$.

\section{A. Filter Analysis}

Theorem 1: Let the filter parameters $G_{i}$ and $K_{i}$ be given. If there exist a sequence of positive scalars $\left\{\mu_{i}>0, i \in \mathcal{S}\right\}$ such that the following $N$ matrix inequalities:

$$
\begin{aligned}
& A_{f i}^{T} P_{i}+P_{i} A_{f i}+\sum_{j=1}^{N} \gamma_{i j} P_{j}+P_{i}\left(A_{f d i} A_{f d i}^{T}+D_{f i} D_{f i}^{T}\right. \\
& \left.+\mu_{i}^{2} M_{f i} M_{f i}^{T}\right) P_{i}+\mu_{i}^{-2} N_{f i}^{T} N_{f i}+F_{f}^{T} H_{i}^{T} H_{i} F_{f}+Q<0
\end{aligned}
$$

have positive definite solutions $P_{i}>0(i \in \mathcal{S})$ and $Q \geq I$, then, the augmented system (16) is exponentially stable in the mean square, independent of the unknown time delay $h$.

Proof: First, we let $C^{2,1}\left(\mathbb{R}^{2 n} \times \mathbb{R}_{+} \times \mathcal{S} ; \mathbb{R}_{+}\right)$denote the family of all nonnegative functions $Y\left(x_{f}, t, i\right)$ on $\mathbb{R}^{2 n} \times \mathbb{R}_{+} \times \mathcal{S}$ which are continuously twice differentiable in $x_{f}$ and differentiable in $t$.

Fix $\xi \in L_{\mathcal{F}_{0}}^{2}\left([-h, 0] ; \mathbb{R}^{2 n}\right)$ arbitrarily and write $x_{f}(t ; \xi)=$ $x_{f}(t)$. Define a Lyapunov functional candidate $Y\left(x_{f}, t, i\right) \in$ $C^{2,1}\left(\mathbb{R}^{2 n} \times \mathbb{R}_{+} \times \mathcal{S} ; \mathbb{R}_{+}\right)$by

$$
\begin{aligned}
Y\left(x_{f}(t), r(t)=i\right): & =Y\left(x_{f}(t), t, i\right) \\
& =x_{f}^{T}(t) P_{i} x_{f}(t)+\int_{t-h}^{t} x_{f}^{T}(s) Q x_{f}(s) d s .
\end{aligned}
$$

It is known (see [7] and [10]) that $\left\{r(t), x_{f}(t)\right\}(t \geq 0)$ is a $C\left([-h, 0] ; \mathbb{R}^{n}\right) \times \mathcal{S}$-valued Markov process. From (16), the weak infinitesimal operator $\mathcal{L}$ of the stochastic process $\left\{r(t), x_{f}(t)\right\}(t \geq 0)$ is given by (20), shown at the bottom of the page.

Note that $\sum_{j=1}^{N} \gamma_{i j}=0$ and, therefore

$$
\begin{aligned}
& \sum_{j=1}^{N} \gamma_{i j} \int_{t-h}^{t} x_{f}^{T}(s) Q x_{f}(s) d s \\
& =\left(\sum_{j=1}^{N} \gamma_{i j}\right)\left(\int_{t-h}^{t} x_{f}^{T}(s) Q x_{f}(s) d s\right) \\
& =0 .
\end{aligned}
$$

Furthermore, since $\Delta A_{f i}(t):=M_{f i} F_{i}(t) N_{f i}$ and $F_{i}^{T}(t) F_{i}(t) \leq I$, it follows from Lemma 1 that, for any scalar $\mu_{i} \neq 0$

$$
\begin{aligned}
P_{i}\left(\Delta A_{f i}(t)\right)+\left(\Delta A_{f i}(t)\right)^{T} P_{i} & \\
& \leq \mu_{i}^{2} P_{i} M_{f i} M_{f i}^{T} P_{i}+\mu_{i}^{-2} N_{f i}^{T} N_{f i} .
\end{aligned}
$$

$$
\begin{aligned}
\mathcal{L} Y\left(x_{f}(t), r(t)\right):= & \lim _{\Delta \rightarrow 0} \frac{1}{\Delta}\left[\mathbb{E}\left\{Y\left(x_{f}(t+\Delta), r(t+\Delta)\right) \mid x_{f}(t), r(t)=i\right\}-Y\left(x_{f}(t), r(t)=i\right)\right] \\
= & x_{f}^{T}(t)\left[\left(A_{f i}+\Delta A_{f i}(t)\right)^{T} P_{i}+P_{i}\left(A_{f i}+\Delta A_{f i}(t)\right)+\sum_{j=1}^{N} \gamma_{i j} P_{j}+Q\right] x_{f}(t) \\
& +x_{f}^{T}(t-h) A_{f d i}^{T} P_{i} x_{f}(t)+x_{f}^{T}(t) P_{i} A_{f d i} x_{f}(t-h) \\
& +x_{f}^{T}(t) P_{i} D_{f i} f\left(F_{f} x_{f}(t, i)\right)+f^{T}\left(F_{f} x_{f}(t, i)\right) D_{f i}^{T} P_{i} x_{f}(t) \\
& -x_{f}^{T}(t-h) Q x_{f}(t-h)+\sum_{j=1}^{N} \gamma_{i j} \int_{t-h}^{t} x_{f}^{T}(s) Q x_{f}(s) d s
\end{aligned}
$$


Moreover, it results from $F_{f} x_{f}(t, i)=x(t, i)$, the inequality (6) and the inequality

$$
\left(f^{T}(x(t, i))-x_{f}^{T}(t) P_{i} D_{f i}\right)\left(f^{T}(x(t, i))-x_{f}^{T}(t) P_{i} D_{f i}\right)^{T} \geq 0
$$

that

$$
\begin{aligned}
& x_{f}^{T}(t) P_{i} D_{f i} f\left(F_{f} x_{f}(t, i)\right)+f^{T}\left(F_{f} x_{f}(t, i)\right) D_{f i}^{T} P_{i} x_{f}(t) \\
\leq & f^{T}(x(t, i)) f(x(t, i))+x_{f}^{T}(t) P_{i} D_{f i} D_{f i}^{T} P_{i} x_{f}(t) \\
\leq & x_{f}^{T}(t)\left(F_{f}^{T} H_{i}^{T} H_{i} F_{f}+P_{i} D_{i} D_{i}^{T} P_{i}\right) x_{f}(t) .
\end{aligned}
$$

Denote

$$
\begin{aligned}
X_{i}:= & A_{f i}^{T} P_{i}+P_{i} A_{f i}+\mu_{i}^{2} P_{i} M_{f i} M_{f i}^{T} P_{i}+\mu_{i}^{-2} N_{f i}^{T} N_{f i} \\
& +\sum_{j=1}^{N} \gamma_{i j} P_{j}+F_{f}^{T} H_{i}^{T} H_{i} F_{f}+P_{i} D_{f i} D_{f i}^{T} P_{i}+Q \\
Z_{i}:= & {\left[\begin{array}{cc}
X_{i} & P_{i} A_{f d i} \\
A_{f d i}^{T} P_{i} & -Q
\end{array}\right] . }
\end{aligned}
$$

Then, substituting (21) and (22) into (20) results in

$$
\begin{aligned}
\mathcal{L} Y\left(x_{f}(t), i\right) \leq & x_{f}^{T}(t) X_{i} x_{f}(t)+x_{f}^{T}(t-h) A_{f d i}^{T} P_{i} x_{f}(t) \\
& +x_{f}^{T}(t) P_{i} A_{f d i} x_{f}(t-h)-x_{f}^{T}(t-h) Q x_{f}(t-h) \\
= & {\left[\begin{array}{ll}
x_{f}^{T}(t) & x_{f}^{T}(t-h)
\end{array}\right] Z_{i}\left[\begin{array}{c}
x_{f}(t) \\
x_{f}(t-h)
\end{array}\right] }
\end{aligned}
$$

From the Schur complement Lemma [3], we know that $Z_{i}<$ 0 if and only if $X_{i}+P_{i} A_{f d i} A_{f d i}^{T} P_{i}<0$, which is the same as the inequality (18). Therefore, we arrive at the conclusion that $\mathcal{L} Y\left(x_{f}(t), i\right)<0$.

Based on the inequality (25), the exponential stability (in the mean square) of the system (16) can be proved as follows by using the techniques developed in [6].

Define

$$
\begin{array}{lc}
\lambda_{P}=\max _{i \in \mathcal{S}} \lambda_{\max }\left(P_{i}\right), & \lambda_{p}=\min _{i \in \mathcal{S}} \lambda_{\min }\left(P_{i}\right) \\
\lambda_{Z}=\min _{i \in \mathcal{S}}\left(-\lambda_{\max }\left(Z_{i}\right)\right), & \lambda_{Q}=\lambda_{\max }(Q)
\end{array}
$$

where $P_{i}>0$ and $Q \geq I$ are solutions to (18), and $Z_{i}$ is defined in (24). Let $\delta$ be the unique root to the equation

$$
\delta\left(\lambda_{P}+h \lambda_{Q} e^{\delta h}\right)=\lambda_{Z}+\min \left(1, \lambda_{Z} e^{\delta h}\right) .
$$

To prove the mean square exponential stability, we modify the Lyapunov function candidate (19) as

$Y_{1}\left(x_{f}(t), t, i\right)=e^{\delta t}\left(x_{f}^{T}(t) P_{i} x_{f}(t)+\int_{t-h}^{t} x_{f}^{T}(s) Q x_{f}(s) d s\right)$ and then, obtain the equation shown at the bottom of the page, where $\mathcal{L} Y\left(x_{f}(s), s, r(s)\right)$ is given in (20) and an upper bound is provided in (25). It follows from (25) that

$$
\begin{aligned}
& \mathbb{E} Y_{1}\left(x_{f}(t), t, r(t)\right) \\
\leq & \left(\lambda_{P}+h\right) \mathbb{E}\|\xi\|^{2}+\left(\delta \lambda_{P}-\lambda_{Z}\right) \mathbb{E} \int_{0}^{t} e^{\delta s}\left|x_{f}(s)\right|^{2} d s \\
& +\delta \mathbb{E} \int_{0}^{t} e^{\delta s} \int_{s-h}^{s} x_{f}^{T}(\alpha) Q x_{f}(\alpha) d \alpha d s \\
& -\lambda_{Z} \mathbb{E} \int_{0}^{t} e^{\delta s}\left|x_{f}(s-h)\right|^{2} d s .
\end{aligned}
$$

Noticing the definition of $\delta$ and the two facts of

$$
\begin{aligned}
& \int_{0}^{t} e^{\delta s} \int_{s-h}^{s} x_{f}^{T}(\alpha) Q x_{f}(\alpha) d \alpha d s \\
\leq & \lambda_{Q} \int_{-h}^{t}\left|x_{f}(\alpha)\right|^{2}\left(\int_{\max (\alpha, 0)}^{\min (\alpha+h, t)} e^{\delta s} d s\right) d \alpha \\
\leq & \lambda_{Q} \int_{-h}^{t}\left|x_{f}(\alpha)\right|^{2} h e^{\delta(\alpha+h)} d \alpha \\
\leq & h \lambda_{Q} e^{\delta h}\left(\frac{\|\xi\|^{2}}{\delta}+\int_{0}^{t} e^{\delta s}\left|x_{f}(s)\right|^{2} d s\right)
\end{aligned}
$$

and

$$
\begin{aligned}
& -\lambda_{Z} \mathbb{E} \int_{0}^{t} e^{\delta s}\left|x_{f}(s-h)\right|^{2} d s \\
= & -\lambda_{Z} e^{\delta h} \mathbb{E} \int_{-h}^{t-h} e^{\delta s}\left|x_{f}(s)\right|^{2} d s \\
\leq & -\min \left(1, \lambda_{Z} e^{\delta h}\right) \mathbb{E} \int_{0}^{t} e^{\delta s}\left|x_{f}(s)\right|^{2} d s \\
& +\mathbb{E} \int_{t-h}^{t} e^{\delta s}\left|x_{f}(s)\right|^{2} d s
\end{aligned}
$$

we have

$\mathbb{E} Y_{1}\left(x_{f}(t), t, r(t)\right) \leq\left(\lambda_{P}+h\left(1+\lambda_{Q} e^{\delta h}\right)\right) \mathbb{E}\|\xi\|^{2}$

$$
+\mathbb{E} \int_{t-h}^{t} e^{\delta s}\left|x_{f}(s)\right|^{2} d s .
$$

Moreover, since $Q \geq I$, we have

$\mathbb{E} Y_{1}\left(x_{f}(t), t, r(t)\right) \geq e^{\delta t} \lambda_{p} \mathbb{E}\left|x_{f}(t)\right|^{2}+\mathbb{E} \int_{t-h}^{t} e^{\delta s}\left|x_{f}(s)\right|^{2} d s$.

It follows from (26)-(28) that $e^{\delta t} \lambda_{p} \mathbb{E}\left|x_{f}(t)\right|^{2} \leq\left(\lambda_{P}+h(1+\right.$ $\left.\left.\lambda_{Q} e^{\delta h}\right)\right) \mathbb{E}\|\xi\|^{2}$, or $\lim _{t \rightarrow \infty} \sup (1 / t) \log \left(\mathbb{E}\left|x_{f}(t, \xi)\right|^{2}\right) \leq-\delta$, which indicates that the trivial solution of (16) is exponentially stable in the mean square. This completes the proof of this theorem.

It can be seen from Theorem 1 that, for a given filter structure, to check the stochastic exponential stability of the system (16), one needs to verify whether there exist scalars $\mu_{i}>0$, matrices $P_{i}>0(i=1,2, \ldots, N)$ and $Q \geq I$ that solve the

$$
\mathbb{E} Y_{1}\left(x_{f}(t), t, r(t)\right)=\mathbb{E} Y_{1}\left(x_{f}(0), 0, r(0)\right)+\mathbb{E} \int_{0}^{t} e^{\delta s}\left[\delta x_{f}^{T}(s) P_{i} x_{f}(s)+\delta \int_{s-h}^{s} x_{f}^{T}(\alpha) Q x_{f}(\alpha) d \alpha+\mathcal{L} Y\left(x_{f}(s), s, r(s)\right)\right] d s
$$


$N$ coupled matrix inequalities. This may be difficult since the $N$ inequalities are coupled and nonlinear on $P_{i}$ and $\mu_{i}$ in their present forms. Fortunately, we can transform them into the associated LMIs [3], and then, we will be able to determine the exponential stability of the system (16) readily by checking the solvability of the LMIs [4]. We restate Theorem 1 in terms of LMIs as follows.

Theorem 2: Let the filter parameters $G_{i}$ and $K_{i}$ be given. If there exist a sequence $\left\{\varepsilon_{i}>0, i \in \mathcal{S}\right\}$, positive definite matrices $P_{i}>0(i \in \mathcal{S})$ and $Q \geq I$ satisfying the following LMIs:

$$
\left[\begin{array}{ccccc}
\Lambda_{i} & P_{i} A_{f d i} & P_{i} D_{f i} & \varepsilon_{i} N_{f i}^{T} & P_{i} M_{f i} \\
A_{f d i}^{T} P_{i} & -I & 0 & 0 & 0 \\
D_{f i}^{T} P_{i} & 0 & -I & 0 & 0 \\
\varepsilon_{i} N_{f i} & 0 & 0 & -\varepsilon_{i} I & 0 \\
M_{f i}^{T} P_{i} & 0 & 0 & 0 & -\varepsilon_{i} I
\end{array}\right]<0
$$

where $\Lambda_{i}$ is defined by $\Lambda_{i}:=A_{f i}^{T} P_{i}+P_{i} A_{f i}+\sum_{j=1}^{N} \gamma_{i j} P_{j}+$ $F_{f}^{T} H_{i}^{T} H_{i} F_{f}+Q$; then, the system (16) is exponentially stable in the mean square.

Proof: Rearrange (18) as

$$
\Lambda_{i}+\left[\begin{array}{llll}
P_{i} A_{f d i} & P_{i} D_{f i} & \mu_{i}^{-1} N_{f i}^{T} & \mu_{i} P_{i} M_{f i}
\end{array}\right]\left[\begin{array}{c}
A_{f d i}^{T} P_{i} \\
D_{f i}^{T} P_{i} \\
\mu_{i}^{-1} N_{f i} \\
\mu_{i} M_{f i}^{T} P_{i}
\end{array}\right]<0
$$

If follows from the Schur complement Lemma ([3]) that, the above inequality holds if and only if

$$
\left[\begin{array}{ccccc}
\Lambda_{i} & P_{i} A_{f d i} & P_{i} D_{f i} & \mu_{i}^{-1} N_{f i}^{T} & \mu_{i} P_{i} M_{f i} \\
A_{f d i}^{T} P_{i} & -I & 0 & 0 & 0 \\
D_{f i}^{T} P_{i} & 0 & -I & 0 & 0 \\
\mu_{i}^{-1} N_{f i} & 0 & 0 & -I & 0 \\
\mu_{i} M_{f i}^{T} P_{i} & 0 & 0 & 0 & -I
\end{array}\right]<0 .
$$

Note that (31) is not linear on $\mu_{i}$. Let $\varepsilon_{i}:=\mu_{i}^{-2}$. Pre- and post-multiplying the inequality (31) by $\operatorname{diag}\left\{I, I, I, \mu_{i}^{-1} I, \mu_{i}^{-1} I\right\}$ yield (29). The proof follows from Theorem 1 immediately.

Remark 3: The inequality (29) is linear on $\varepsilon_{i}>0, P_{i}>0$ $(i=1,2, \ldots, N)$ and $Q \geq I$, and thus standard LMI techniques [3], [4] can be exploited to check the exponential stability of the closed-loop system (16) when the filter is given.

\section{B. Filter Design}

The following lemma, which is easily accessible, will be used in the proofs of our main results in this paper.

Lemma 2: For a given negative definite matrix $\Upsilon<0(\Upsilon \in$ $\left.\mathbb{R}^{n \times n}\right)$, there always exists a matrix $S \in \mathbb{R}^{n \times p}(p \leq n)$ such that $\Upsilon+S S^{T}<0$.

For the sake of simplicity, we give the following definitions:

$$
\begin{aligned}
\widehat{A}_{i} & :=A_{i}+\left(A_{d i} A_{d i}^{T}+D_{i} D_{i}^{T}+\mu_{i}^{2} M_{1 i} M_{1 i}^{T}\right) P_{1 i} \\
\widehat{C}_{i} & :=C_{i}+\mu_{i}^{2} M_{2 i} M_{1 i}^{T} P_{1 i} \\
R_{i} & :=\mu_{i}^{2} M_{2 i} M_{2 i}^{T}, \quad \Theta_{i}:=\widehat{C}_{i}+\mu_{i}^{2} M_{2 i} M_{1 i}^{T} P_{2 i} .
\end{aligned}
$$

The following theorem establishes an approach to designing the desired filter parameters in terms of the positive definite solutions to two sets of matrix inequalities.

Theorem 3: If there exist a sequence of positive scalars $\left\{\mu_{i}>0, i \in \mathcal{S}\right\}$ such that the following two sets of matrix inequalities:

$$
\begin{aligned}
A_{i}^{T} P_{1 i} & +P_{1 i} A_{i}+\sum_{j=1}^{N} \gamma_{i j} P_{1 j}+P_{1 i}\left(A_{d i} A_{d i}^{T}+D_{i} D_{i}^{T}\right. \\
& \left.+\mu_{i}^{2} M_{1 i} M_{1 i}^{T}\right) P_{1 i}+\mu_{i}^{-2} N_{i}^{T} N_{i}+H_{i}^{T} H_{i}+Q_{1}<0 \\
\Upsilon_{i}:= & \left(\widehat{A}_{i}-\mu_{i}^{2} M_{1 i} M_{2 i}^{T} R_{i}^{-1} \widehat{C}_{i}\right)^{T} P_{2 i} \\
& +P_{2 i}\left(\widehat{A}_{i}-\mu_{i}^{2} M_{1 i} M_{2 i}^{T} R_{i}^{-1} \widehat{C}_{i}\right) \\
& +\sum_{j=1}^{N} \gamma_{i j} P_{2 j}+P_{2 i}\left(A_{d i} A_{d i}^{T}+D_{i} D_{i}^{T}+\mu_{i}^{2} M_{1 i} M_{1 i}^{T}\right. \\
& \left.-\mu_{i}^{4} M_{1 i} M_{2 i}^{T} R_{i}^{-1} M_{2 i} M_{1 i}^{T}\right) P_{2 i}-\widehat{C}_{i}^{T} R_{i}^{-1} \widehat{C}_{i}+Q_{2}<0
\end{aligned}
$$

have positive definite solutions $P_{1 i}>0, P_{2 i}>0(i \in \mathcal{S})$, $Q_{1} \geq I$, and $Q_{2} \geq I$, where the matrices $\widehat{A}_{i}, \widehat{C}_{i}, R_{i}$ are defined, respectively, in (32)-(33); then, the filter (7) with parameters

$$
\begin{aligned}
K_{i} & =P_{2 i}^{-1}\left[\Theta_{i}^{T} R_{i}^{-1}+S_{i} U_{i} R_{i}^{-1 / 2}\right] \\
G_{i} & =\widehat{A}_{i}-K_{i} \widehat{C}_{i}
\end{aligned}
$$

where $\Theta_{i}$ is defined in (33), $U_{i} \in \mathbb{R}^{p \times p}$ is arbitrary orthogonal (i.e., $\left.U_{i} U_{i}^{T}=I\right), S_{i} \in \mathbb{R}^{n \times p}$ is an arbitrary matrix meeting $\Upsilon_{i}+S_{i} S_{i}^{T}<0$ and $\Upsilon_{i}$ is defined in (35), will be such that the augmented system (16) is exponentially stable in the mean square for all admissible parameter uncertainties $\Delta A_{i}(t), \Delta C_{i}(t)$ and the nonlinear disturbance input $f(x(t, i))$, independent of the unknown time-delay $h$.

Proof: First, we define

$$
\begin{aligned}
\Sigma_{i}:= & A_{f i}^{T} P_{i}+P_{i} A_{f i}+\sum_{j=1}^{N} \gamma_{i j} P_{j}+P_{i}\left(A_{f d i} A_{f d i}^{T}+D_{f i} D_{f i}^{T}\right. \\
& \left.+\mu_{i}^{2} M_{f i} M_{f i}^{T}\right) P_{i}+\mu_{i}^{-2} N_{f i}^{T} N_{f i}+F_{f}^{T} H_{i}^{T} H_{i} F_{f}+Q \\
:= & {\left[\begin{array}{ll}
\Sigma_{11 i} & \Sigma_{12 i} \\
\Sigma_{12 i}^{T} & \Sigma_{22 i}
\end{array}\right] . }
\end{aligned}
$$

Then, by setting $P_{i}=\operatorname{diag}\left\{P_{1 i}, P_{2 i}\right\}>0$ and $Q=\operatorname{diag}\left\{Q_{1}, Q_{2}\right\}>0$, we have

$$
\begin{aligned}
\Sigma_{11 i}= & A_{i}^{T} P_{1 i}+P_{1 i} A_{i}+\sum_{j=1}^{N} \gamma_{i j} P_{1 j}+P_{1 i}\left(A_{d i} A_{d i}^{T}+D_{i} D_{i}^{T}\right. \\
& \left.+\mu_{i}^{2} M_{1 i} M_{1 i}^{T}\right) P_{1 i}+\mu_{i}^{-2} N_{i}^{T} N_{i}+H_{i}^{T} H_{i}+Q_{1} \\
\Sigma_{12 i}= & \left(A_{i}-G_{i}-K_{i} C_{i}\right)^{T} P_{2 i} \\
& +P_{1 i}\left[A_{d i} A_{d i}^{T}+D_{i} D_{i}^{T}+\mu_{i}^{2} M_{1 i}\left(M_{1 i}-K_{i} M_{2 i}\right)^{T}\right] P_{2 i}
\end{aligned}
$$

$$
\begin{aligned}
\Sigma_{22 i}= & G_{i}^{T} P_{2 i}+P_{2 i} G_{i}+\sum_{j=1}^{N} \gamma_{i j} P_{2 j}+P_{2 i}\left[A_{d i} A_{d i}^{T}+D_{i} D_{i}^{T}\right. \\
& \left.+\mu_{i}^{2}\left(M_{1 i}-K_{i} M_{2 i}\right)\left(M_{1 i}-K_{i} M_{2 i}\right)^{T}\right] P_{2 i}+Q_{2} .
\end{aligned}
$$


It follows directly from (34) that $\Sigma_{11 i}<0$. Also, Assumption 2 ensures $R_{i}^{-1}>0$ exists. In the light of $G_{i}=\widehat{A}_{i}-K_{i} \widehat{C}_{i}$ and the definitions of $R_{i}$ and $\Theta_{i}$, we obtain

$$
\begin{aligned}
& \Sigma_{22 i} \\
= & \widehat{A}_{i}^{T} P_{2 i}+P_{2 i} \widehat{A}_{i}+\sum_{j=1}^{N} \gamma_{i j} P_{2 j} \\
& +P_{2 i}\left(A_{d i} A_{d i}^{T}+D_{i} D_{i}^{T}+\mu_{i}^{2} M_{1 i} M_{1 i}^{T}\right) P_{2 i} \\
& -P_{2 i} K_{i} \Theta_{i}-\Theta_{i}^{T} K_{i}^{T} P_{2 i}+\left(P_{2 i} K_{i}\right) R_{i}\left(P_{2 i} K_{i}\right)^{T}+Q_{2} \\
= & \widehat{A}_{i}^{T} P_{2 i}+P_{2 i} \widehat{A}_{i}+\sum_{j=1}^{N} \gamma_{i j} P_{2 j}+P_{2 i}\left(A_{d i} A_{d i}^{T}\right. \\
& \left.+D_{i} D_{i}^{T}+\mu_{i}^{2} M_{1 i} M_{1 i}^{T}\right) P_{2 i}-\Theta_{i}^{T} R_{i}^{-1} \Theta_{i} \\
& +\left[\left(P_{2 i} K_{i}\right) R_{i}^{1 / 2}-\Theta_{i}^{T} R_{i}^{-1 / 2}\right]\left[\left(P_{2 i} K_{i}\right) R_{i}^{1 / 2}-\Theta_{i}^{T} R_{i}^{-1 / 2}\right]^{T}+Q_{2} .
\end{aligned}
$$

By utilizing (36) and the orthogonality of $U_{i}$, we can see that

$$
\begin{array}{r}
{\left[\left(P_{2 i} K_{i}\right) R_{i}^{1 / 2}-\Theta_{i}^{T} R_{i}^{-1 / 2}\right]\left[\left(P_{2 i} K_{i}\right) R_{i}^{1 / 2}-\Theta_{i}^{T} R_{i}^{-1 / 2}\right]^{T}} \\
=\left(S_{i} U_{i}\right)\left(S_{i} U_{i}\right)^{T}=S_{i} S_{i}^{T}
\end{array}
$$

and therefore, it follows from the definition of $\Theta_{i}$ in (33) that

$$
\begin{aligned}
\Sigma_{22 i}= & \left(\widehat{A}_{i}-\mu_{i}^{2} M_{1 i} M_{2 i}^{T} R_{i}^{-1} \widehat{C}_{i}\right)^{T} P_{2 i} \\
& +P_{2 i}\left(\widehat{A}_{i}-\mu_{i}^{2} M_{1 i} M_{2 i}^{T} R_{i}^{-1} \widehat{C}_{i}\right)+\sum_{j=1}^{N} \gamma_{i j} P_{2 j} \\
& +P_{2 i}\left(A_{d i} A_{d i}^{T}+D_{i} D_{i}^{T}+\mu_{i}^{2} M_{1 i} M_{1 i}^{T}\right. \\
& \left.-\mu_{i}^{4} M_{1 i} M_{2 i}^{T} R_{i}^{-1} M_{2 i} M_{1 i}^{T}\right) P_{2 i} \\
& -\widehat{C}_{i}^{T} R_{i}^{-1} \widehat{C}_{i}+Q_{2}+S_{i} S_{i}^{T} \\
= & \Upsilon_{i}+S_{i} S_{i}^{T}
\end{aligned}
$$

where $\Upsilon_{i}$ is defined in (35). Since $S_{i} \in \mathbb{R}^{n \times p}$ is an arbitrary matrix meeting $\Upsilon_{i}+S_{i} S_{i}^{T}<0$, the conclusion $\Sigma_{22 i}<0$ follows from (35) easily.

Furthermore, substituting (37) into (40) immediately yields $\Sigma_{12 i}=0$, and therefore we have the conclusion that $\Sigma_{i}<0$. Finally, it follows from Theorem 1 that the augmented system (16) is exponentially stable in the mean square for all admissible parameter uncertainties $\Delta A_{i}(t), \Delta C_{i}(t)$ and the nonlinear disturbance input $f(x(t, i))$, independent of the unknown time-delay $h$. This proves Theorem 2 .

Remark 4: Theorem 3 gives a QMI approach to the design of robust filters for a class of uncertain time-delay jump systems with nonlinear disturbances. The solvability of the coupled QMIs (34) and (35) plays a crucial role in the filter design. Fortunately, the parameters $P_{2 i}(i \in \mathcal{S})$ of (35) are not included in (34). Thus, we may first solve (34) for $\mu_{i}>0, P_{1 i}>0(i \in \mathcal{S})$ and $Q_{1} \geq I$. Then, the inequalities (35) are coupled QMIs for $P_{2 i}(i \in \mathcal{S})$, and can be solved by the generalized matrix Riccati inequality/equation approach (see [1]). It remains to focus on the algorithm for solving (34). Similar to Theorem 2, it is easy to convert (34) into the following LMIs:

$$
\left[\begin{array}{ccccc}
\Xi_{i} & P_{1 i} A_{d i} & P_{1 i} D_{i} & \varepsilon_{i} N_{i}^{T} & P_{1 i} M_{1 i} \\
A_{d i}^{T} P_{1 i} & -I & 0 & 0 & 0 \\
D_{i}^{T} P_{1 i} & 0 & -I & 0 & 0 \\
\varepsilon_{i} N_{i} & 0 & 0 & -\varepsilon_{i} I & 0 \\
M_{1 i}^{T} P_{1 i} & 0 & 0 & 0 & -\varepsilon_{i} I
\end{array}\right]<0
$$

where $\Xi_{i}:=A_{i}^{T} P_{1 i}+P_{1 i} A_{i}+\sum_{j=1}^{N} \gamma_{i j} P_{1 j}+H_{i}^{T} H_{i}+Q_{1}$, and $\varepsilon_{i}:=\mu_{i}^{-2}$. Since the inequality (45) is linear on $\varepsilon$ and $P_{1 i}>0$ $(i=1,2, \ldots, N)$, we can employ the standard LMI techniques [3], [4] to check the solvability of the original matrix inequality (34).

\section{A NumericAl EXAMPLE}

We assume that the system (1)-(3) has two modes and the data are as follows:

$$
\begin{aligned}
& A_{1}=\left[\begin{array}{cc}
-3.52 & 0.06 \\
0.05 & -3.75
\end{array}\right] \\
& A_{2}=\left[\begin{array}{ll}
-3.18 & -0.06 \\
-0.05 & -4.05
\end{array}\right] \\
& A_{d 1}=0.1 I_{2} \\
& A_{d 2}=-0.1 I_{2} \\
& D_{1}=0.2 I_{2} \\
& D_{2}=-0.2 I_{2} \\
& C_{1}=0.95 I_{2} \\
& C_{2}=0.98 I_{2} \\
& H_{1}=0.1 I_{2} \\
& H_{2}=0.1 I_{2} \\
& M_{11}=0.3 I_{2} \\
& M_{12}=-0.3 I_{2} \\
& M_{21}=-0.45 I_{2} \\
& M_{22}=0.18 I_{2} \\
& N_{1}=0.4 I_{2} \\
& N_{2}=-0.4 I_{2} \\
& \Pi=\left[\begin{array}{cc}
-3 & 3 \\
0.4 & -0.4
\end{array}\right] \\
& f(x(t, 1))=f(x(t, 2))=\left[\begin{array}{l}
0.1 \sin x_{1}(t) \\
0.1 \sin x_{2}(t)
\end{array}\right] \\
& F(t, 1)=F(t, 2)=\sin t I_{2} \\
& h=0.1 \\
& \varphi(t)=0.1 \text {. }
\end{aligned}
$$

Letting $Q_{1}=Q_{2}=I$ and solving the LMIs (45) ( $i=1$, 2) by using the LMI toolbox [4], we obtain that $\varepsilon_{1}=4.2060$, $\varepsilon_{2}=3.0553$, and

$P_{11}=\left[\begin{array}{cc}97.0583 & -1.5593 \\ -1.5593 & 103.6028\end{array}\right] P_{12}=\left[\begin{array}{cc}78.3547 & 1.3921 \\ 1.3921 & 100.6307\end{array}\right]$

and therefore $\mu_{1}=0.4876$ and $\mu_{2}=0.5721$. Next, solve the QMIs (35) $(i=1,2)$ to give

$$
P_{21}=\left[\begin{array}{cc}
19.2754 & -0.6180 \\
-0.6180 & 21.8746
\end{array}\right] \quad P_{22}=\left[\begin{array}{ll}
1.1425 & 0.4754 \\
0.4754 & 8.8454
\end{array}\right] \text {. }
$$


We first consider the system mode 1 . In this case, we choose $S_{1}$ (meeting $\Upsilon_{1}+S_{1} S_{1}^{T}<0$ ) and the orthogonal matrix $U_{1}$ as $S_{1}=4 I_{2}, U_{1}=\operatorname{diag}(1,-1)$, and then it follows from (36)-(37) that

$K_{1}=\left[\begin{array}{cc}-2.0538 & -0.0451 \\ 0.0083 & -3.7567\end{array}\right] \quad G_{1}=\left[\begin{array}{cc}-1.0351 & -0.0558 \\ 0.1447 & -5.2768\end{array}\right]$.

Now, let us consider the system mode 2 . We set $S_{2}$ (meeting $\Upsilon_{2}+S_{2} S_{2}^{T}<0$ ) and the orthogonal matrix $U_{2}$ as $S_{2}=I_{2}$ and $U_{2}=I_{2}$, and then we have

$$
K_{2}=\left[\begin{array}{cc}
-27.0405 & 1.0789 \\
1.1014 & -9.1401
\end{array}\right] G_{2}=\left[\begin{array}{cc}
-7.8750 & 0.2469 \\
0.2816 & -3.3260
\end{array}\right] \text {. }
$$

\section{CONCLUSION}

We have studied the problem of robust filtering for a class of uncertain time-delay systems with Markovian jumping parameters and nonlinear disturbances. A linear filter has been designed to achieve the prescribed robust exponential stability constraints (in the mean square), regardless of the admissible parameter uncertainties, the bounded nonlinear disturbance input and the unknown state delay. Both the filter analysis and design issues have been discussed in detail by means of LMIs. We have derived both the existence conditions and the analytical expression of desired filters. It has been emphasized that, using the developed approach, the expected robust exponential filters, when they exist, are usually a large set, and the remaining freedom can be used to meet other desired performance requirements. We may also extend our results to the discrete-time case. The results will appear in the near future.

\section{REFERENCES}

[1] H. Abou-Kandil, G. Freiling, and G. Jank, "Solution and asymptotic behavior of coupled Riccati equations in jump linear systems," IEEE Trans. Automat. Contr., vol. 39, pp. 1631-1636, Aug. 1994.

[2] E.-K Boukas and Z.-K. Liu, Deterministic and Stochastic Time-Delay Systems. Boston, MA: Birkhauser, 2002.

[3] S. Boyd, L. El Ghaoui, E. Feron, and V. Balakrishnan, "Linear matrix inequalities in system and control theory," in Studies in Applied Mathematics. Philadelphia, PA: SIAM, 1994.

[4] P. Gahinet, A. Nemirovsky, A. J. Laub, and M. Chilali, LMI Control Toolbox: For Use with Matlab. Natick, MA: MathWorks Inc., 1995.

[5] M. S. Mahmoud, Robust Control and Filtering for Time-Delay Systems. New York: Dekker, 2000.

[6] X. Mao, "Stability of stochastic differential equations with Markovian switching," Stoch. Processes Applicat., vol. 79, pp. 45-67, 1999.

[7] — "Exponential stability of stochastic delay interval systems with Markovian switching," IEEE Trans. Automat. Contr, vol. 47, pp. 1604-1612, Oct. 2002.

[8] S.-I. Niculescu, E. I. Verriest, L. Dugard, and J. M. Dion, "Stability and robust stability of time-delay systems: A guided tour," in Stability and Control of Time-Delay Systems, L. Dugard et al., Eds. Berlin, Germany: Springer-Verlag, 1998, vol. 228, pp. 1-71.

[9] A. Saberi, P. Sannuti, and B. M. Chen, $\mathrm{H}_{2}$ Optimal Control. London, U.K.: Prentice-Hall, 1995.

[10] A. V. Skorohod, Asymptotic Methods in the Theory of Stochastic Differential Equations. Providence, RI: Amer. Math. Soc., 1989.

[11] P. Shi, E. K. Boukas, and R. K. Agarwal, "Kalman filtering for continuous-time uncertain systems with Markovian jumping parameters," IEEE Trans. Automat. Contr., vol. 44, pp. 1592-1597, Aug. 1999.

[12] Z. Wang and K. J. Burnham, "Robust filtering for a class of stochastic uncertain nonlinear time-delay systems via exponential state estimation," IEEE Trans. Signal Processing, vol. 49, pp. 794-804, 2001. 